\title{
Los morteros del Convento de Santa Paula (Sevilla)
}

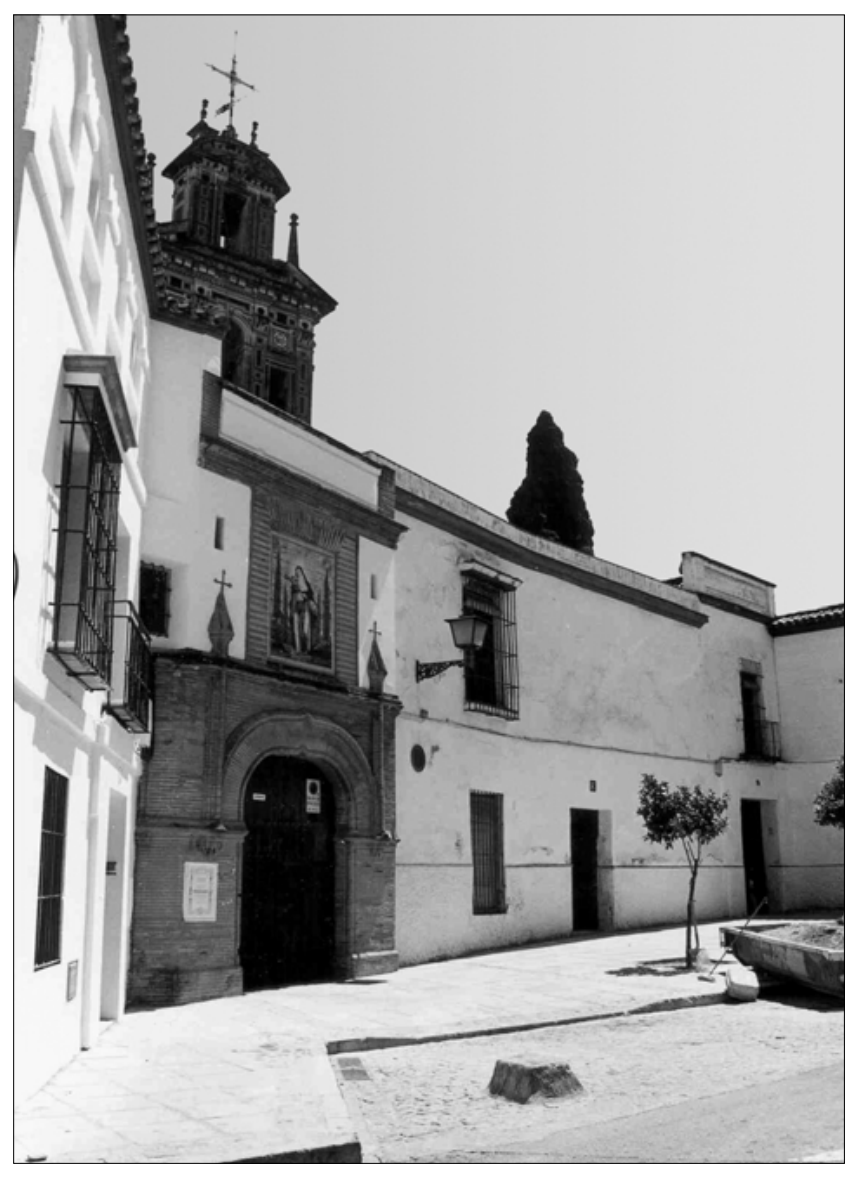

Fig I. Fachada del Convento de Sta. Paula desde la calle Sta. Paula
Fco. Javier Alejandre Sánchez

Profesor Asociado de Materiales / E.U. de Arquitectura Técnica Universidad de Sevilla

Emilio Minguito Sarrión

Jefe Sección Obras de Rehabilitación de la Gerencia de Urbanismo Ayuntamiento de Sevilla

Juan Jesús Martín del Río

Colaborador Honorario Dpto. de Construcciones Arquitectónicas II

Universidad de Sevilla

\section{Palabras clave}

Convento de Santa Paula / revestimientos / morteros / cal / fibras vegetales

\section{Resumen}

El estudio de los materiales constitutivos de una obra o edificación, además de ser una de las etapas que permite la recopilación de información en el análisis de su situación, debe tener una gran influencia en la posterior toma de decisiones sobre las actuaciones que se van a realizar sobre ella.

En esta ocasión, dentro del proyecto de intervención de la Miras del Convento de Santa Paula (Sevilla), se ha llevado a cabo la caracterización de los morteros mediante el análisis de su composición y dosificación, porosidad, adherencia, y mediante la utilización de la lupa binocular.

Los resultados obtenidos muestran en general el empleo de morteros con elevados contenidos en cal y abundante presencia de fibras de origen vegetal. Esta información ha sido de utilidad para la elaboración de los morteros de reposición de los revestimientos con mayor deterioro. 
$\longleftarrow$
$\square$

Fig 2. Plano de situación de Convento de Sta. Paula (Díaz Chamorro, 1960)

Fig 3. Vista de las miras del Convento de Sta. Paula desde uno de sus claustros

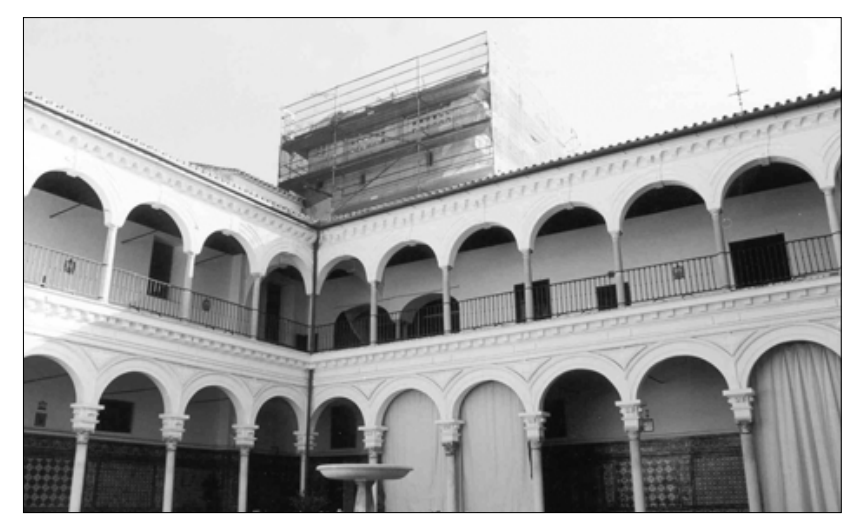

\section{Historia}

El Convento de Sta. Paula fue fundado de la mano de Dña. Ana Fernández de Santillán y Guzmán, que a la muerte de su esposo e hija, decide recluirse en el emparedamiento de San Juan de la Palma, desde donde solicitó la bula de fundación del Convento que le será concedida en el I 473 por Sixto IV.

En los años sucesivos se bendicen diferentes casas en el barrio de San Román propiedad de Dña. Ana que irán formando el primer núcleo conventual. Figura importante a reseñar es Dña. Isabel Enríquez, marquesa de Montemayor y amiga de la fundadora, que fue la principal artífice de la erección de la iglesia del Convento.
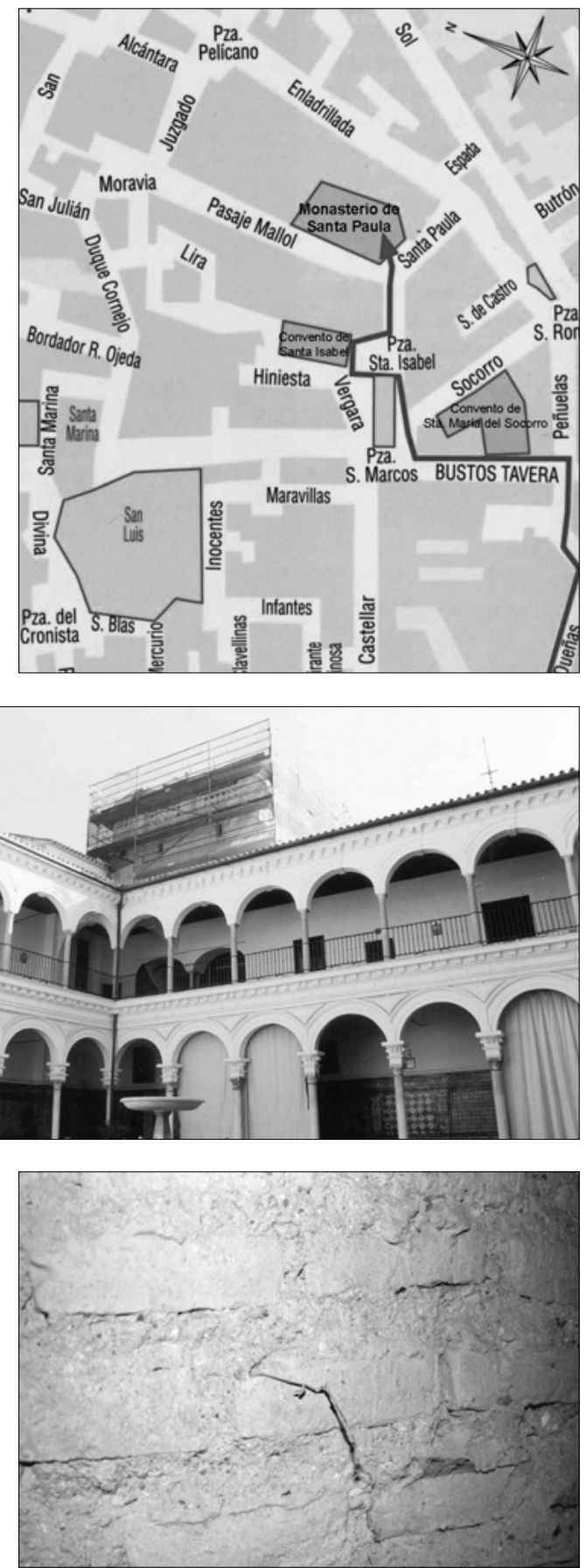

A lo largo de los siglos y hasta nuestros días diferentes construcciones del Convento han sufrido una serie de restauraciones, reformas, rehabilitaciones y modificaciones que están estrechamente relacionadas con el objeto de este trabajo:

- Entre 1615 y 1620 se amplia el Convento, concretamente se realiza el ala del claustro medianero a la iglesia y la escalera, así como, la construcción del mirador, que se encuentra situado en el claustro mayor o "patio nuevo" adosado a la Iglesia, concretamente en el ala norte de dicho claustro sobre la escalera principal.

- En 1858 existe una reparación en todo el recinto por el arquitecto D. Manuel Portillo y Navarrete.

- Por último, cabe destacar las obras que se realizan en las miras entre 1958-1960 para reforzar las vigas del forjado, trabajo realizado por D. Francisco Martínez.

El convento de Santa Paula está declarado BIC (BOE de 4 de Junio de 1931) de conformidad con la Ley de Protección del Patrimonio Histórico Español de 1985. Así mismo, está catalogado A (Protección Integral) en el Plan General de Ordenación Urbana de Sevilla de 1987.

\section{La intervención}

El proyecto de intervención sobre las miras del Convento de Santa Paula se encuentra enmarcado dentro de los programas plurianuales del Servicio de Rehabilitación y Conservación del Patrimonio de la Gerencia de Urbanismo del Ayuntamiento de Sevilla. En su memoria se recogen como actuaciones principales: la restauración de la armadura de cubierta, del forjado y del artesonado de la escalera principal (figura 3). Además de ellas, se plantea la restauración de los revestimientos exteriores e interiores, intervención que ha dado origen al siguiente estudio para caracterizar los morteros que los constituyen.

\section{Descripción de las muestras}

Se han estudiado un total de ocho muestras de morteros, cuatro pertenecientes al exterior de las miras, y cuatro al interior de las mismas. Sus códigos de designación y sus funciones constructivas se muestran en la tabla I.

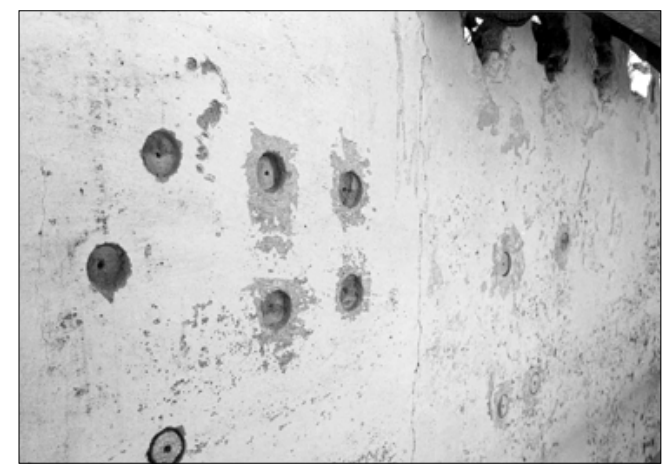




\section{Análisis de dosificaciones}

El análisis de dosificaciones de los morteros se ha realizado mediante el ataque con ácido clorhídrico $(I: I)$, según el método de Jedrzejewska, que permite la estimación del contenido de carbonato cálcico (NLT-I 19/72) y de arena presente en un mortero de cal. Estos dos parámetros obtenidos tienen validez para aproximar la dosificación original del mortero siempre que la arena empleada en su confección sea mayoritariamente de naturaleza silícea-silicatada.

Se ha analizado también el contenido de sulfato (expresado como $\mathrm{SO}_{3}$ ) según UNE |46500, siendo posible de esta manera valorar la presencia de yeso en el mortero, bien como conglomerante o como producto de la alteración del mismo.

Los resultados de los análisis así como la composición y dosificación de morteros de referencia se exponen en la tabla 2.

Se puede observar como en las muestras MI, RI, $\mathrm{Rm}, \mathrm{M} 2$, E2 y $\mathrm{R} 2$, el contenido de $\mathrm{CaCO}_{3}$ ha sido muy elevado (50,4\%-67,2\%). De este intervalo de valores se puede concluir al ser las arenas de naturaleza silicea-silicatada, que la mayoría del carbonato proviene de la carbonatación de la cal, siendo por lo tanto los morteros originarios muy ricos en cal, con dosificaciones en ésta muy superiores a la $1: 1$, pudiéndose incluso llegar a hablar de "cales con muchas impurezas".

La muestra $\mathrm{EI}$ ha sido la que ha tenido un menor contenido de $\mathrm{CaCO}_{3}$ (21,8\%), a excepción de Mr, por lo que estamos ante un mortero de cal con dosificación I:I.

En cuanto al contenido de sulfatos $\left(\mathrm{SO}_{3}\right)$ se pueden establecer tres grupos de muestras: el primero, para las muestras MI, RI y M2 cuyos valores han sido bajos (0,21\%-0,25\%), el segundo, para las muestras EI, Rm, E2 y R2 con valores medios ( $1,12 \%-3.23 \%$ ), y el tercero, para la muestra Mr que ha sido muy alto $(31,64 \%)$.

El origen del $\mathrm{SO}_{3}$ para el primer grupo al ser muy escaso está muy probablemente asociado a las materias primas; para el segundo grupo, además del posible origen natural se podría especular con la adición de pequeñas cantidades de yeso al mortero de cal para mejorar determinadas propiedades de éste (rapidez de fraguado y resistencias mecánicas iniciales) (Dorrego et al., 1998; Robador González et al., 1998). Por último, el mortero Mr es un yeso con impurezas de calcita, sílice y silicatos.

\section{Porosidad}

Como es sabido, la medida de la porosidad de un mortero y en general de cualquier material, se puede realizar por distintos métodos: inmersión en agua, la porosimetría por inyección de mercurio, la adsor-
Tabla 1. Designación y funciones constructivas de los morteros

\begin{tabular}{|c|c|c|}
\hline LOCALIZACION & DESIGNACIÓN & FUNCIÓN CONSTRUCTIVA \\
\hline \multirow{4}{*}{ Exterior de las miras } & M1 & Mortero de fábrica de los pilares \\
\hline & E1 & Enfoscado exterior de los pilares \\
\hline & R1 & $\begin{array}{l}\text { Revoco exterior sobre tabique de } \\
\text { cerramiento de miras }\end{array}$ \\
\hline & $\mathrm{Rm}$ & $\begin{array}{l}\text { Revoco sobre mochetas de los } \\
\text { pilares }\end{array}$ \\
\hline \multirow{4}{*}{ Interior de las miras } & M2 & $\begin{array}{l}\text { Mortero de fábrica de muros } \\
\text { interiores de las miras }\end{array}$ \\
\hline & E2 & Enfoscado interior sobre muros \\
\hline & $\mathrm{R} 2$ & Revoco interior sobre E2 \\
\hline & $\mathrm{Mr}$ & $\begin{array}{l}\text { Mortero de relleno y revestimien } \\
\text { to en las cabezas de las vigas }\end{array}$ \\
\hline
\end{tabular}

Tabla 2. Composición de los morteros

\begin{tabular}{|c|c|c|c|c|c|c|}
\hline \multicolumn{2}{|c|}{ Muestras } & \multicolumn{2}{|c|}{ Árido $\mathrm{CaCO}_{3}$ Fracción } & $\mathrm{SO}_{3}(\%)$ & \multirow{2}{*}{ soluble } & \\
\hline & & Naturaleza & Contenido & & & \\
\hline \multirow{4}{*}{$\begin{array}{l}\text { Exterior de } \\
\text { la miras }\end{array}$} & M1 & Silícea-silicatada * & 49,4 & 50,4 & 0,2 & 0,25 \\
\hline & E1 & Silícea-silicatada & 71,1 & 21,8 & 7,1 & 3,23 \\
\hline & $\mathrm{R} 1$ & Silícea & 26,6 & 54,8 & 18,6 & 0,25 \\
\hline & $\mathrm{Rm}$ & Silícea & 22,0 & 67,2 & 10,8 & 1,73 \\
\hline \multirow{4}{*}{$\begin{array}{l}\text { Interior de } \\
\text { las miras }\end{array}$} & M2 & Silícea-silicatada & 32,0 & 53,0 & 15,0 & 0,21 \\
\hline & E2 & Silícea-silicatada & 30,7 & 58,4 & 10,9 & 1,12 \\
\hline & $\mathrm{R} 2$ & Silícea & 19,3 & 63,7 & 17,0 & 3,05 \\
\hline & $\mathrm{Mr}$ & Silícea-silicatada & 23,0 & 21,0 & 56,0 & 31,64 \\
\hline \multicolumn{2}{|c|}{$\begin{array}{c}\text { Morteros referencia } \\
1: 1 \text { (cal:arena) }\end{array}$} & & 79 & 21 & - & - \\
\hline \multirow{2}{*}{\multicolumn{2}{|c|}{$\begin{array}{l}\text { 1:2 (cal:arena) } \\
\text { 1:3 (cal:arena) }\end{array}$}} & silícea & 85 & 15 & - & - \\
\hline & & & 89 & 11 & - & - \\
\hline
\end{tabular}

* Naturaleza mayoritaria silícea

ción de nitrógeno y el análisis de imagen al microscopio. Cada uno de ellos proporciona una determinada información, abarcando desde la porosidad total, el diámetro de los poros, la distribución de poros por diámetro, el volumen porcentual de cada diámetro, hasta la porosidad cerrada o inaccesible. Ninguno es excluyente respecto a los demás, y normalmente ofrecen datos complementarios, al tener parcialmente solapados sus rangos de aplicación.

De todos ellos se ha aplicado para esta investigación el de absorción de agua (saturando el material a ebullición), que sirve para cuantificar el sistema poroso de los morteros respecto a su interacción con el agua.

Los valores de porosidad accesible al agua, son por tanto, un criterio de valoración de la calidad y el estado de conservación de los morteros (tabla 3). 


\begin{tabular}{|c|c|c|c|}
\hline & \multicolumn{3}{|c|}{ Tabla 3. Relación calidad vs porosidad en los morteros } \\
\hline morteros & $\begin{array}{c}\text { buena calidad } \\
\text { compacto }\end{array}$ & $\begin{array}{c}\text { buenos pero } \\
\text { porosos }\end{array}$ & $\begin{array}{l}\text { baja calidad } \\
\text { muy porosos }\end{array}$ \\
\hline Porosidad & $<20 \%$ & $20-30 \%$ & $>30 \%$ \\
\hline
\end{tabular}

Los resultados obtenidos para los morteros se muestran en la siguiente tabla:

Tabla 4. Porosidad abierta de los morteros

\begin{tabular}{cc} 
MUESTRAS & POROSIDAD ABIERTA (\%) \\
\hline MI & 44,7 \\
\hline EI & 35,4 \\
\hline RI & 42,3 \\
\hline Rm & 53,8 \\
\hline M2 & 55,9 \\
\hline E2 & 54,5 \\
\hline R2 & 37,4 \\
\hline Mr & 44,7 \\
\hline $\begin{array}{c}\text { Mortero referencia } \\
\text { ::3 (cal:arena) }\end{array}$ \\
\hline
\end{tabular}

Todas las muestras han tenido unos valores de porosidad abierta muy elevados (35,4\%-55,9\%), por lo que se pueden clasificar como morteros muy porosos. El origen de esta alta porosidad se debe probablemente a la utilización de grandes cantidades de agua en el amasado del mortero para conferirle mayor plasticidad y trabajabilidad, agua que por evaporación se elimina en su mayoría generando la porosidad.

\section{Adherencia}

La adherencia se ha ensayado según la Norma UNE 83-822-95/Exp. (figura 5), y los valores obtenidos de-

Figura 5. Ensayo de adherencia sobre el revoco R2

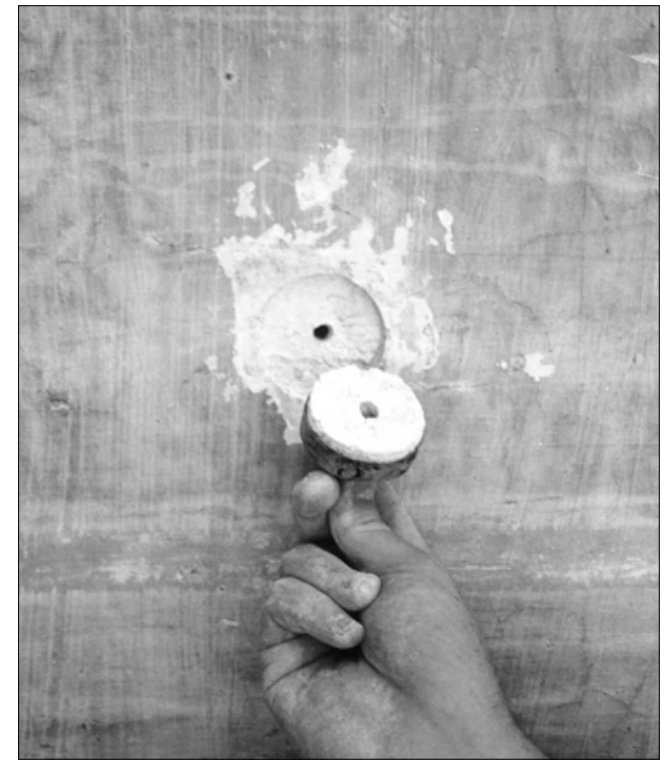

penden de múltiples factores: clase de mortero (tipo de conglomerante, relación agua/conglomerante y dosificación), clase de soporte, de la mano de obra en la ejecución, aunque son también factores a considerar las condiciones de curado, el grado de humidificación del soporte, la edad, etc.

La falta de adherencia de los morteros para fábricas va a perjudicar a la estabilidad y solidez de la misma, y en los morteros de revestimiento, origina en una primera etapa al desprenderse parcialmente del soporte el abolsamiento o abofamiento, y finalmente, el desprendimiento total del revestimiento respecto del soporte.

Los resultados del ensayo de adherencia de los morteros se exponen en la tabla 5.

Tabla 5. Adherencia de algunos morteros de revestimiento

\begin{tabular}{cc} 
MUESTRAS & $\begin{array}{c}\text { ADHERENCIA } \\
\left(\mathrm{Kg} / \mathrm{cm}^{2}\right)\end{array}$ \\
\hline $\mathrm{EI}$ & 0,3 \\
\hline $\mathrm{RI}$ & 2,4 \\
\hline $\mathrm{R} 2$ & ${ }^{\mathrm{a}} 0,0$ \\
\hline Morteros referencia & \\
$\mathrm{I}: 3$ (cal:arena) & $3, \mathrm{I}$ (a 90 días) \\
$\mathrm{I}: 4$ (cal:arena) & 2,0 (a 90 días) \\
\hline
\end{tabular}

Los valores de adherencia obtenidos han sido muy dispares, oscilando entre resultados muy buenos, como el caso del revoco $\mathrm{RI}\left(2,4 \mathrm{Kg} / \mathrm{cm}^{2}\right)$, y resultados prácticamente nulos como R2 $\left(0,0 \mathrm{Kg} / \mathrm{cm}^{2}\right)$. La muestra $\mathrm{EI}$ ha tenido un adherencia baja, de 0,3 $\mathrm{Kg} / \mathrm{cm}^{2}$.

La justificación de estos resultados resulta compleja teniendo en cuenta los múltiples factores que pueden influir en la adherencia. Una hipótesis posible para el revoco R2 es la existencia de filtraciones de agua procedentes de la cubierta, y que han podido disolver parcialmente la interfase de unión con el soporte. En el caso del enfoscado El puede tener influencia que sea el de más bajo contenido en cal.

\section{Estudio con lupa binocular}

Las muestras de morteros se han observado en un microscopio óptico Nikon Stereoscopic SMZ-2T a un aumento 45X. A continuación se comentan las fotos más significativas:

En figura 6 se puede observar la estructura estratigráfica del revestimiento interior. Destaca la el color blanco del mismo y que es debido a la abundancia de cal de ambos morteros. En el enfoscado E2 se puede apreciar la presencia de una partícula de carbón, que es una impureza procedente del horno de calcinación de la cal. El espesor medio del encalado es pequeño, solo de $110 \mathrm{~mm}$, y corresponde a unas 4-5 capas de cal. 
Las figuras 7 y 8 confirman la frecuente presencia de fibras vegetales en casi todos los morteros estudiados (figura 3). Teniendo en cuenta la abundancia de estas fibras, más que pensar en una impureza, se puede especular con la adición intencionada de las mismas para mejorar el comportamiento de los morteros (disminución de la fisuración por retracción y aumento de la resistencia a flexión). La adición de pelos y fibras vegetales (paja, barcia, etc.) como fibra de refuerzo en los morteros es una práctica habitual desde tiempos prehistóricos (Alvarez et al., 1995; Alejandre, 1998 y 2000).

\section{Morteros de reposición}

Los morteros empleados para la sustitución o reposición de los originales más deteriorados (tanto exte-

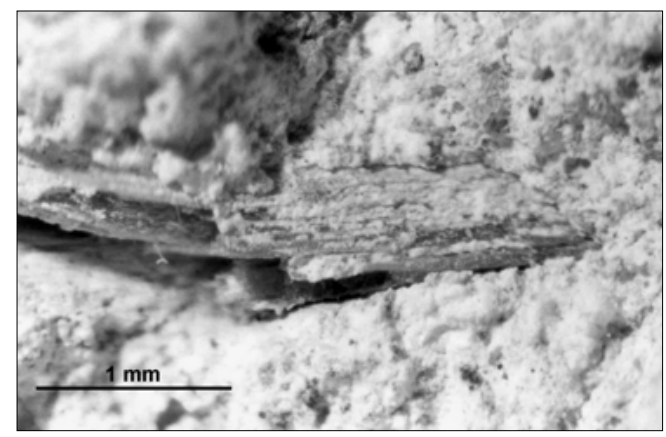

riores como interiores), han sido para los enfoscados morteros de cal de dosificación 1:2 y con mezcla al $50 \%$ de marmolina y arena de río, y morteros de cal de dosificación I: I, para los revocos (figura 9). En los revestimientos originales bien conservados, se ha procedido al raspado del encalado, y a la aplicación posterior de un enjalbegado.

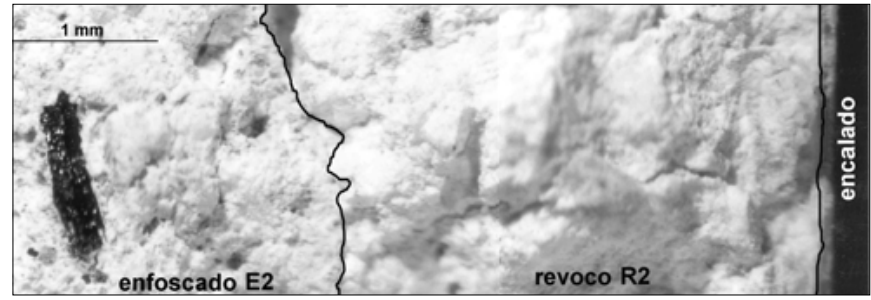

Figura 6. Estructura completa del revestimiento exterior $\varangle$

느

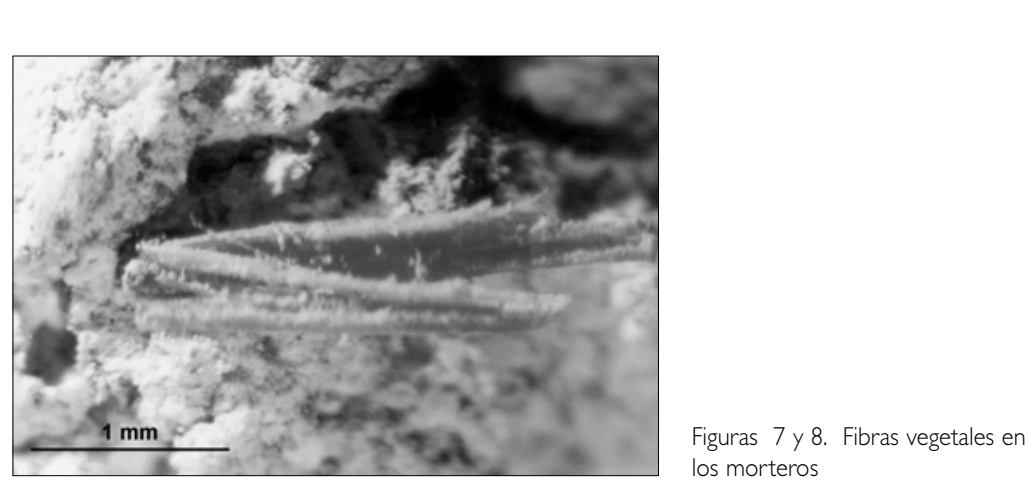

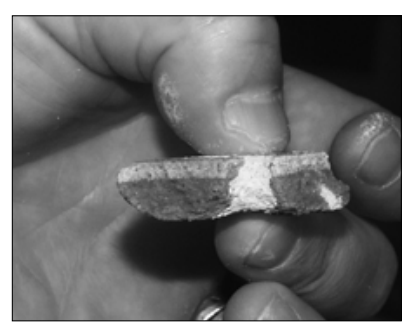

Figura 9. Izda. Estructura del revestimiento de reposición (enfoscado y revoco).

Dcha. Test de la fenolftaleina (Peroni et al,, 1981) para controlar la evolución de la carbonatación
AENOR. Norma UNE 146500. Determinación del contenido de compuestos de azufre en los áridos por métodos gravimétricos e instrumentales. Madrid, 1998.

AENOR. Norma UNE 83822 EX. Morteros. Métodos de ensayo. morteros endurecidos. determinación de la adherencia de los morteros de revoco y enlucido. Madrid, 1995.

ALEJANDRE SÁNCHEZ, F.J. Los Morteros En La Antigüedad. Capítulo de libro en: "La Técnica De La Arquitectura En La Antigüedad", Edit. A. Graciani, 1998, 79-96 pp.

ALEJANDRE SÁNCHEZ, F.J. Los Morteros En La Edad Media. Cap. de libro en: "La Técnica De La Arquitectura En La Edad Media", Edit. A. Graciani, 200, 235-250 pp.

ÁlVAREZ GALINDO, J.l.; MARTíN PÉREZ, A.; GARCÍA CASADO, P.j. Historia de los morteros. PH: Boletín Informativo del I.A.P.H., n 13, Sevilla, 1995, 52-59.

CEDEX. NLT- I I6. Carbonatos en los suelos por el método del calcímetro de Bernard. Madrid, 1972.
DÍAZ CHAMORRO, V. Itinerarios por los Conventos de Sevilla. Sevilla: Area de Economía y Turismo del Ayuntamiento de Sevilla, 1996.

DORREGO, F.; LUXÁN, M.P.; SOTOLONGO, R. Los trabadillos: origen, utilización y técnicas de preparación. Actas del $2^{\circ}$ Congreso Nacional de $\mathrm{H}^{\mathrm{a}}$ de la Construcción. A Coruña, 22-24 de Octubre de 1998, |45-150 pp.

PERONI, S.; TERSIGNI, G.; TORRACA, G.; CEREA, S.; FORTI, M.; GUIDOBALDI, F.; ROSSI-DORIA, P.; DE REGE, A.; PICCHI, F..;; PIETRAFITTA, G.; BENEDETTI, G. Lime based mortars for the repair of ancient masonry and possible substitutes. Mortars, Cements and Grouts used in Conservation of Historic Buldings. Symposium 3-6. I I. Roma., 1981.

ROBADOR GONZÁLEZ, M.D.; LINARES DÍAZ, J.F. El revestimiento de la piedra en la Catedral de Burgos. Actas del $2^{\circ}$ Congreso Nacional de $H^{a}$ de la Construcción. A Coruña, 22-24 de Octubre de 1998, 4I 7-429 pp. 